\title{
AN OPTIMAL DIFFERENTIABLE SPHERE THEOREM FOR COMPLETE MANIFOLDS
}

\author{
Hong-Wei Xu and Juan-Ru Gu
}

\begin{abstract}
Dedicated to Professor S.-S. Chern on his 100th anniversary
Abstract. A new differentiable sphere theorem is obtained from the view of submanifold geometry. We introduce a new scalar quantity involving both the scalar curvature and the mean curvature of an oriented complete submanifold $M^{n}$ in a space form $F^{n+p}(c)$ with $c \geq 0$. Making use of the convergence results of Hamilton and Brendle for Ricci flow and the Lawson-Simons formula for the nonexistence of stable currents, we prove that if the infimum of this scalar quantity is positive, then $M$ is diffeomorphic to $S^{n}$. We then introduce an intrinsic invariant $I(M)$ for oriented complete Riemannian $n$-manifold $M$ via the scalar quantity, and prove that if $I(M)>0$, then $M$ is diffeomorphic to $S^{n}$. It should be emphasized that our differentiable sphere theorem is optimal for arbitrary $n(\geq 2)$. Moreover, we generalize the Brendle-Schoen differentiable sphere theorem for manifolds with strictly $1 / 4$-pinched curvatures in the pointwise sense to the cases of submanifolds in a Riemannian manifold with codimension $p(\geq 0)$.
\end{abstract}

\section{Introduction}

The investigation of curvature and topology of Riemannian manifolds or submanifolds is one of the main stream in global differential geometry. In 1897, Hadamard [12] proved a classical sphere theorem which says that any oriented compact surface with positive Gaussian curvature in $R^{3}$ must be diffeomorphic to a sphere. It was seen from the Gauss-Bonnet-Chern theorem $[1,10]$ that Hadamard's sphere theorem could be extended to the cases of compact Riemannian surfaces with positive curvature. A natural problem is stated as follows.

Problem 1.1. Is it possible to generalize the Hadamard sphere theorem for compact Riemannian surfaces to higher dimensional cases?

In 1951, Rauch [19] first proved a topological sphere theorem for positive pinched compact manifolds. During the past six decades, there are many important progresses on topological and differentiable pinching problems for Riemannian manifolds. The most famous topological sphere theorem is Berger-Klingenberg's quarter pinching theorem, which has been improved by many geometers [3, 6, 20, 26]. Recently Brendle and Schoen [8] obtained a classification of compact and simply connected manifolds

Received by the editors February 26, 2010.

2000 Mathematics Subject Classification. 53C20; 53C40.

Keywords: Complete manifolds, differentiable sphere theorem, Ricci flow, stable currents, second fundamental form.

Research supported by the NSFC, Grant No. 10771187, 11071211; the Trans-Century Training Programme Foundation for Talents by the Ministry of Education of China. 
with weakly 1/4-pinched curvatures. Consequently, they obtained the following striking result.

Theorem A. Let $M$ be an $n$-dimensional complete and simply connected Riemannian manifold such that $1 / 4 \leq K_{M} \leq 1$. Then $M$ is either diffeomorphic to $S^{n}$, or isometric to a compact rank one symmetric space (CROSS).

Since the dimension of a complex projective space is always even, Brendle and Schoen's differentiable sphere theorem is optimal for even dimensional cases. More recently Petersen and Tao [17] have improved Brendle and Schoen's pinching constant in Theorem A to $\frac{1}{4}-\varepsilon_{n}$, where $\varepsilon_{n}$ is a positive constant depending only on $n$. However, Petersen and Tao's pinching constant is not yet optimal for odd dimensional cases. In fact, the constant $\varepsilon_{n}$ is not explicit.

The following important convergence result for the Ricci flow, initiated by Brendle and Schoen [7], was finally obtained by Brendle [4].

Theorem B. Let $\left(M, g_{0}\right)$ be a compact Riemannian manifold of dimension $n(\geq 4)$. Assume that

$$
R_{1313}+\lambda^{2} R_{1414}+R_{2323}+\lambda^{2} R_{2424}-2 \lambda R_{1234}>0
$$

for all orthonormal four-frames $\left\{e_{1}, e_{2}, e_{3}, e_{4}\right\}$ and all $\lambda \in[-1,1]$. Then the normalized Ricci flow with initial metric $g_{0}$

$$
\frac{\partial}{\partial t} g(t)=-2 R i c_{g(t)}+\frac{2}{n} r_{g(t)} g(t)
$$

exists for all time and converges to a constant curvature metric as $t \rightarrow \infty$. Here $r_{g(t)}$ denotes the mean value of the scalar curvature of $g(t)$.

Let $M^{n}$ be an $n(\geq 2)$-dimensional submanifold in an $(n+p)$-dimensional Riemannian manifolds $N^{n}+p$. Denote by $H$ and $S$ the mean curvature and the squared length of the second fundamental form of $M$ respectively. Using nonexistence for stable currents on compact submanifolds of a sphere and the generalized Poincare conjecture for dimension $n(\geq 5)$ proved by Smale, Lawson and Simons [16] proved that if $M^{n}(n \geq 5)$ is an oriented compact submanifold in $S^{n+p}$, and if $S<2 \sqrt{n-1}$, then $M$ is homeomorphic to a sphere.

Let $F^{n+p}(c)$ be an $(n+p)$-dimensional simply connected space form with nonnegative constant curvature $c$. Putting

$$
\alpha(n, H, c)=n c+\frac{n^{3}}{2(n-1)} H^{2}-\frac{n(n-2)}{2(n-1)} \sqrt{n^{2} H^{4}+4(n-1) c H^{2}},
$$

we have $\min _{H} \alpha(n, H, c)=2 \sqrt{n-1} c$. Motivated by a rigidity theorem in [23, 24], Shiohama and $\mathrm{Xu}$ [21] improved Lawson-Simons' result and proved the following topological sphere theorem.

Theorem C. Let $M^{n}(n \geq 4)$ be an oriented complete submanifold in $F^{n+p}(c)$ with $c \geq 0$. Suppose that $\sup _{M}(S-\alpha(n, H, c))<0$. Then $M$ is homeomorphic to a sphere.

The following differentiable sphere theorem for hypersurfaces follows from the 
convergence results for the mean curvature flow and parabolic flow due to Huisken [14] and Andrews [2], respectively.

Theorem D. Let $M^{n}$ be an n-dimensional oriented closed hypersurface in $F^{n+1}(c)$ with $c>0$. If $S<2 c+\frac{n^{2} H^{2}}{n-1}$, then $M$ is diffeomorphic to $S^{n}$.

Recently, Xu and Zhao [25] proved a differentiable sphere theorem for submanifolds of a sphere with codimension $p$.

Theorem E. Let $M^{n}$ be an $n(\geq 4)$-dimensional oriented complete submanifold in $F^{n+p}(c)$ with $c>0$. Then

(i) if $4 \leq n \leq 6$ and $\sup _{M} S<2 \sqrt{n-1} c$, then $M$ is diffeomorphic to $S^{n}$,

(ii) if $n \geq 7$ and $S<2 \sqrt{2} c$, then $M$ is diffeomorphic to $S^{n}$.

Motivated by Theorems C, D and E, we propose the following differentiable pinching problem.

Problem 1.2. Let $M^{n}$ be an oriented complete submanifold in $F^{n+p}(c)$ with $c \geq 0$. Suppose that $\sup _{M}\left(S-\frac{n^{2} H^{2}}{n-1}-2 c\right)<0$. Is it possible to prove that $M$ must be diffeomorphic to $S^{n}$ ?

Since any Riemannian manifold can be considered as a submanifold in the same manifold with codimension zero, the following problem seems attractive.

Problem 1.3. Is it possible to generalize the Brendle-Schoen differentiable sphere theorem [7] for manifolds with strictly 1/4-pinched curvatures in the pointwise sense to the cases of submanifolds in a Riemannian manifold with codimension $p(\geq 0)$ ?

The purpose of the present article is to solve Problems 1.1, 1.2 and 1.3, and prove some new differentiable pinching theorems for complete submanifolds and Riemannian manifolds via Ricci flow and stable currents. More precisely, we obtain the following results.

Theorem 1.1. Let $M^{n}$ be an $n$-dimensional complete submanifold in an $(n+p)$ dimensional Riemannian manifold $N^{n+p}$. Denote by $\bar{K}(\pi)$ the sectional curvature of $N$ for tangent 2-plane $\pi\left(\subset T_{x} N\right)$ at point $x \in N$. Set $\bar{K}_{\max }:=\max _{\pi \subset T_{x} N} \bar{K}(\pi)$, $\bar{K}_{\min }:=\min _{\pi \subset T_{x} N} \bar{K}(\pi)$. If $S<\frac{8}{3}\left(\bar{K}_{\min }-\frac{1}{4} \bar{K}_{\max }\right)+\frac{n^{2} H^{2}}{n-1}$, then $M$ is diffeomorphic to a spherical space form or $R^{n}$. In particular, if $M$ is simply connected, then $M$ is diffeomorphic to $S^{n}$ or $R^{n}$.

Remark 1.1. When $M$ is a compact submanifold of codimension zero, Theorem 1.1 reduces to the differentiable pinching theorem of Brendle and Schoen [7].

Theorem 1.2. Let $M^{n}$ be an n-dimensional oriented complete submanifold in 
$F^{n+p}(c)$ with $c \geq 0$. If

$$
\lambda(M):=\sup _{M}\left(S-\frac{n^{2} H^{2}}{n-1}-2 c\right)<0,
$$

then $M$ is diffeomorphic to $S^{n}$.

We shall show in Example 4.1 that Theorem 1.2 is optimal for arbitrary $n(\geq 2)$ and $p$. It follows from the Gauss equation that the pinching condition in Theorem 1.2 is equivalent to

$$
\mu(M):=-\lambda(M)=\inf _{M}\left[R-\frac{n^{2}(n-2)}{n-1} H^{2}-(n+1)(n-2) c\right]>0,
$$

where $R$ is the scalar curvature of $M$.

For a complete Riemannian $n$-manifold $M^{n}$, we set $\mathcal{C}:=\left\{\varphi ; \varphi: M \longrightarrow F^{n+p}(c)\right.$ is an isometric embedding for some constant $c \geq 0$ and positive integer $p\}$ and $\mathcal{D}:=\left\{\varphi ; \varphi: M \longrightarrow R^{n+p}\right.$ is an isometric embedding for some positive integer $\left.p\right\}$. With the aid of the Nash embedding theorem, we get $\mathcal{C} \supset \mathcal{D} \neq \emptyset$. We define two intrinsic invariants $I(M)$ and $I_{0}(M)$ by

$$
\begin{aligned}
I(M):= & \sup _{\varphi \in \mathcal{C}} \mu(M, \varphi):=\sup _{\varphi \in \mathcal{C}} \inf _{M}\left[R-\frac{n^{2}(n-2)}{n-1} H^{2}-(n+1)(n-2) c\right], \\
& I_{0}(M):=\sup _{\varphi \in \mathcal{D}} \mu(M, \varphi):=\sup _{\varphi \in \mathcal{D}} \inf _{M}\left[R-\frac{n^{2}(n-2)}{n-1} H^{2}\right] .
\end{aligned}
$$

Notice that $I(M) \geq I_{0}(M)$. We shall prove

Theorem 1.3. Let $M^{n}$ be an oriented complete Riemannian n-manifold. If $I(M)>$ 0 , then $M$ is diffeomorphic to $S^{n}$. In particular, if $I_{0}(M)>0$, then $M$ is diffeomorphic to $S^{n}$.

Remark 1.2. In the case $n=2$, Theorem 1.3 reduces to the Hadamard sphere theorem for compact Riemannian surfaces. We shall give an example (Example 4.2) to show that our differentiable sphere theorem for Riemannian manifolds is optimal for arbitrary $n(\geq 2)$.

Furthermore, we obtain some other differentiable pinching theorems for complete submanifolds in Riemannian manifolds, which extend the sphere theorems due to Huisken, $\mathrm{Xu}$ and Zhao [14, 25].

\section{Preliminaries}

Let $M^{n}$ be an $n(\geq 2)$-dimensional submanifold in an $(n+p)$-dimensional Riemannian manifolds $N^{n+p}$. We shall make use of the following convention on the range of indices.

$$
1 \leq A, B, C, \ldots \leq n+p ; 1 \leq i, j, k, \ldots \leq n ; n+1 \leq \alpha, \beta, \gamma, \ldots \leq n+p .
$$

For an arbitrary fixed point $x \in M \subset N$, we choose an orthonormal local frame field $\left\{e_{A}\right\}$ in $N^{n+p}$ such that $e_{i}$ 's are tangent to $M$. Denote by $\left\{\omega_{A}\right\}$ the dual frame 
field of $\left\{e_{A}\right\}$. Let $R m$ and $\overline{R m}$ be the Riemannian curvature tensors of $M$ and $N$ respectively, and $h$ the second fundamental form of $M$. Then

$$
\begin{aligned}
& R m=\sum_{i, j, k, l} R_{i j k l} \omega_{i} \otimes \omega_{j} \otimes \omega_{k} \otimes \omega_{l}, \\
& \overline{R m}=\sum_{A, B, C, D} \bar{R}_{A B C D} \omega_{A} \otimes \omega_{B} \otimes \omega_{C} \otimes \omega_{D}, \\
& h=\sum_{\alpha, i, j} h_{i j}^{\alpha} \omega_{i} \otimes \omega_{j} \otimes e_{\alpha}, \\
& R_{i j k l}=\bar{R}_{i j k l}+\sum_{\alpha}\left(h_{i k}^{\alpha} h_{j l}^{\alpha}-h_{i l}^{\alpha} h_{j k}^{\alpha}\right) .
\end{aligned}
$$

The squared norm $S$ of the second fundamental form and the mean curvature $H$ of $M$ are given by

$$
S:=\sum_{\alpha, i, j}\left(h_{i j}^{\alpha}\right)^{2}, H:=\left|\frac{1}{n} \sum_{\alpha, i} h_{i i}^{\alpha} e_{\alpha}\right| .
$$

Denote by $K(\pi)$ the sectional curvature of $M$ for tangent 2-plane $\pi\left(\subset T_{x} M\right)$ at point $x \in M, \bar{K}(\pi)$ the sectional curvature of $N$ for tangent 2-plane $\pi\left(\subset T_{x} N\right)$ at point $x \in N$. Set $\bar{K}_{\min }:=\min _{\pi \subset T_{x} N} \bar{K}(\pi), \bar{K}_{\max }:=\max _{\pi \subset T_{x} N} \bar{K}(\pi)$. Then by Berger's inequality (See e.g. [5], Proposition 1.9), we have

$$
\left|\bar{R}_{A B C D}\right| \leq \frac{2}{3}\left(\bar{K}_{\max }-\bar{K}_{\min }\right)
$$

for all distinct indices $A, B, C, D$.

The following nonexistence theorem for stable currents in a compact Riemannian manifold $M$ isometrically immersed into $F^{n+p}(c)$ is employed to eliminate the homology groups $H_{q}(M ; Z)$ for $0<q<n$, which was initiated by Lawson-Simons [16] and extended by Xin [22].

Theorem 2.1. Let $M^{n}$ be a compact submanifold in $F^{n+p}(c)$ with $c \geq 0$. Assume that

$$
\sum_{k=q+1}^{n} \sum_{i=1}^{q}\left[2\left|h\left(e_{i}, e_{k}\right)\right|^{2}-\left\langle h\left(e_{i}, e_{i}\right), h\left(e_{k}, e_{k}\right)\right\rangle\right]<q(n-q) c
$$

holds for any orthonormal basis $\left\{e_{i}\right\}$ of $M_{x}$ at any point $x \in M$, where $q$ is an integer satisfying $0<q<n$. Then there does not exist any stable q-currents. Moreover,

$$
H_{q}(M ; Z)=H_{n-q}(M ; Z)=0,
$$

where $H_{i}(M ; Z)$ is the $i$-th homology group of $M$ with integer coefficients.

Corollary 2.1. Let $M^{n}$ be a compact submanifold in $F^{n+p}(c)$ with $c \geq 0$. Assume that

$$
\sum_{k=2}^{n}\left[2\left|h\left(e_{1}, e_{k}\right)\right|^{2}-\left\langle h\left(e_{1}, e_{1}\right), h\left(e_{k}, e_{k}\right)\right\rangle\right]<(n-1) c
$$

holds for any orthonormal basis $\left\{e_{i}\right\}$ of $M_{x}$ at any point $x \in M$. Then $\pi_{1}(M)=0$. 
Proof. Suppose that $\pi_{1}(M) \neq 0$. Since $M$ is compact, it follows from a classical theorem due to Cartan and Hadamard that there exists a minimal closed geodesic in any non-trivial homotopy class in $\pi_{1}(M)$. However, by Theorem 2.1 we know that there does not exist any stable integral currents on $M$. This contradicts the hypothesis. Therefore, $\pi_{1}(M)=0$. This completes the proof.

\section{Sphere theorem in dimension three}

We first prove the following sphere theorem for submanifolds of dimension three.

Theorem 3.1. Let $M^{3}$ be a 3-dimensional compact submanifold in a simply connected space form $F^{3+p}(c)$ with nonnegative constant curvature c. If $S<2 c+\frac{9}{2} H^{2}$, then $M$ is diffeomorphic to $S^{3}$.

Proof. Notice that

$$
\begin{aligned}
& \sum_{k=q+1}^{3} \sum_{i=1}^{q}\left[2\left|h\left(e_{i}, e_{k}\right)\right|^{2}-\left\langle h\left(e_{i}, e_{i}\right), h\left(e_{k}, e_{k}\right)\right\rangle\right] \\
= & 2 \sum_{\alpha} \sum_{k=q+1}^{3} \sum_{i=1}^{q}\left(h_{i k}^{\alpha}\right)^{2}-\sum_{\alpha} \sum_{k=q+1}^{3} \sum_{i=1}^{q} h_{i i}^{\alpha} h_{k k}^{\alpha} \\
= & \sum_{\alpha}\left[2 \sum_{k=q+1}^{3} \sum_{i=1}^{q}\left(h_{i k}^{\alpha}\right)^{2}-\left(\sum_{i=1}^{q} h_{i i}^{\alpha}\right)\left(\sum_{i=1}^{3} h_{i i}^{\alpha}-\sum_{i=1}^{q} h_{i i}^{\alpha}\right)\right] .
\end{aligned}
$$

Putting

$$
S_{\alpha}:=\sum_{i, j=1}^{3}\left(h_{i j}^{\alpha}\right)^{2}, \quad T_{\alpha}:=\sum_{i=1}^{3} h_{i i}^{\alpha}, \quad \tilde{S}_{\alpha}:=\sum_{i=1}^{3}\left(h_{i i}^{\alpha}\right)^{2}
$$

we get

$$
S=\sum_{\alpha} S_{\alpha}, \quad 9 H^{2}=\sum_{\alpha} T_{\alpha}^{2}
$$

and

$$
\begin{aligned}
q r \tilde{S}_{\alpha} & =q r \sum_{i=1}^{q}\left(h_{i i}^{\alpha}\right)^{2}+q r \sum_{k=q+1}^{3}\left(h_{k k}^{\alpha}\right)^{2} \\
& \geq r\left(\sum_{i=1}^{q} h_{i i}^{\alpha}\right)^{2}+q\left(\sum_{k=q+1}^{3} h_{k k}^{\alpha}\right)^{2}
\end{aligned}
$$

where $r:=3-q$. Substituting

$$
T_{\alpha}-\sum_{i=1}^{q} h_{i i}^{\alpha}=\sum_{k=q+1}^{3} h_{k k}^{\alpha}
$$

into (4), we obtain

$$
3\left(\sum_{i=1}^{q} h_{i i}^{\alpha}\right)^{2}-2 q T_{\alpha} \sum_{i=1}^{q} h_{i i}^{\alpha}+q T_{\alpha}^{2}-q r \tilde{S}_{\alpha} \leq 0 .
$$


Set

$$
Z_{\alpha}:=-\left(\sum_{i=1}^{q} h_{i i}^{\alpha}\right)\left(T_{\alpha}-\sum_{i=1}^{q} h_{i i}^{\alpha}\right)
$$

It follows from (5) that

$$
3 Z_{\alpha}+(r-q) T_{\alpha} \sum_{i=1}^{q} h_{i i}^{\alpha}+q T_{\alpha}^{2}-q r \tilde{S}_{\alpha} \leq 0 .
$$

Making use of the equalities

$$
\begin{aligned}
& \sum_{i=1}^{3}\left(h_{i i}^{\alpha}-\frac{T_{\alpha}}{3}\right)=0, \quad \sum_{i=1}^{3}\left(h_{i i}^{\alpha}-\frac{T_{\alpha}}{3}\right)^{2}=\tilde{S}_{\alpha}-\frac{T_{\alpha}^{2}}{3}, \\
& \sum_{i=1}^{q}\left(h_{i i}^{\alpha}-\frac{T_{\alpha}}{3}\right)+\frac{q}{3} T_{\alpha}=\sum_{i=1}^{q} h_{i i}^{\alpha},
\end{aligned}
$$

we obtain

$$
\tilde{S}_{\alpha}-\frac{T_{\alpha}^{2}}{3} \geq\left(\frac{1}{q}+\frac{1}{r}\right)\left[\sum_{i=1}^{q}\left(h_{i i}^{\alpha}-\frac{T_{\alpha}}{3}\right)\right]^{2} .
$$

So, we get

$$
\left|\sum_{i=1}^{q}\left(h_{i i}^{\alpha}-\frac{T_{\alpha}}{3}\right)\right| \leq \sqrt{\frac{q r}{3}\left(\tilde{S}_{\alpha}-\frac{T_{\alpha}^{2}}{3}\right)} .
$$

This together with (6) implies

$$
Z_{\alpha} \leq \frac{q r}{3} \tilde{S}_{\alpha}-\left[\frac{q(r-q)}{9}+\frac{q}{3}\right] T_{\alpha}^{2}+\frac{|r-q|}{3}\left|T_{\alpha}\right| \sqrt{\frac{q r}{3}\left(\tilde{S}_{\alpha}-\frac{T_{\alpha}^{2}}{3}\right)} .
$$

It follows from (3), (9) and the relations $q r=2$ and $|r-q|=1$ that

$$
\begin{aligned}
& \sum_{k=q+1}^{3} \sum_{i=1}^{q}\left[2\left|h\left(e_{i}, e_{k}\right)\right|^{2}-\left\langle h\left(e_{i}, e_{i}\right), h\left(e_{k}, e_{k}\right)\right\rangle\right]-q r c \\
\leq & \sum_{\alpha}\left[S_{\alpha}-\frac{\tilde{S}_{\alpha}}{3}-\frac{4}{9} T_{\alpha}^{2}+\frac{\left|T_{\alpha}\right|}{3} \sqrt{\frac{2}{3}\left(\tilde{S}_{\alpha}-\frac{T_{\alpha}^{2}}{3}\right)}\right]-2 c \\
\leq & S-4 H^{2}-2 c-\frac{1}{3} \sum_{\alpha} \tilde{S}_{\alpha}+\sum_{\alpha}\left[\frac{T_{\alpha}^{2}}{18}+\frac{1}{3}\left(\tilde{S}_{\alpha}-\frac{T_{\alpha}^{2}}{3}\right)\right] \\
= & S-\frac{9}{2} H^{2}-2 c .
\end{aligned}
$$

Then under the assumption, we obtain

$$
\sum_{k=q+1}^{3} \sum_{i=1}^{q}\left[2\left|h\left(e_{i}, e_{k}\right)\right|^{2}-\left\langle h\left(e_{i}, e_{i}\right), h\left(e_{k}, e_{k}\right)\right\rangle\right]-q r c<0 .
$$


This together with Corollary 2.1 implies that $\pi_{1}(M)=0$.

It follows from Proposition 2.1 in [21] and the assumption for $S$ that

$$
\begin{aligned}
\operatorname{Ric}_{M}(X) & \geq \frac{2}{3}\left[3 c+6 H^{2}-S-\frac{3}{\sqrt{6}} H\left(S-3 H^{2}\right)^{1 / 2}\right] \\
& =\frac{2}{3}\left[\left(3 c+\frac{27}{4} H^{2}-\frac{3}{2} S\right)+\frac{3}{4} H^{2}+\frac{1}{2}\left(S-3 H^{2}\right)-\frac{3}{\sqrt{6}} H\left(S-3 H^{2}\right)^{1 / 2}\right] \\
& >0
\end{aligned}
$$

holds for any unit vector $X \in T_{x} M$ at each point $x \in M$. From Hamilton's convergence result for Ricci flow in three dimensions [13], it follows that $M$ is diffeomorphic to a 3-dimensional spherical space form. This completes the proof of Theorem 3.1.

Corollary 3.1. Let $M$ be a 3-dimensional compact submanifold in the unit sphere $S^{3+p}$. Suppose that $H \geq \frac{2}{3} \sqrt{\sqrt{2}-1}$. If $S<2 \sqrt{2}$, then $M$ is diffeomorphic to $S^{3}$.

Proof. By a direct computation, we get

$$
S<2+\frac{9}{2} H^{2} .
$$

By Theorem 3.1, we conclude that $M$ is diffeomorphic to $S^{3}$. This proves Corollary 3.1 .

Up to now, the following problem proposed by Lawson and Simons [16] is still open.

Problem 3.1. Let $M$ be a 3-dimensional oriented compact submanifold in the unit sphere $S^{3+p}$. Suppose that $S<2 \sqrt{2}$. Can one prove that $M$ must be diffeomorphic to $S^{3}$ ?

\section{Differentiable sphere theorem in higher dimensions}

The following lemma will be used in the proof of our differentiable pinching theorems.

Lemma 4.1. Let $M^{n}$ be an $n$-dimensional submanifold in an $(n+p)$-dimensional Riemannian manifold $N^{n+p}$, and $\pi$ a tangent 2-plane on $T_{x} M$ at point $x \in M$. Choose an orthonormal two-frame $\left\{e_{1}, e_{2}\right\}$ at $x$ such that $\pi=\operatorname{span}\left\{e_{1}, e_{2}\right\}$. Then

$$
K(\pi) \geq \frac{1}{2}\left(2 \bar{K}_{\min }+\frac{n^{2} H^{2}}{n-1}-S\right)+\sum_{\alpha=n+1}^{n+p} \sum_{j>i,(i, j) \neq(1,2)}\left(h_{i j}^{\alpha}\right)^{2} .
$$

Proof. We extend the orthonormal two-frame $\left\{e_{1}, e_{2}\right\}$ to $\left\{e_{1}, \cdots, e_{n+p}\right\}$ such that $e_{i}$ 's are tangent to $M$. Setting $S_{\alpha}:=\sum_{i, j=1}^{n}\left(h_{i j}^{\alpha}\right)^{2}$, we have

$$
\left(\sum_{i=1}^{n} h_{i i}^{\alpha}\right)^{2}=(n-1)\left[\sum_{i=1}^{n}\left(h_{i i}^{\alpha}\right)^{2}+\sum_{i \neq j}\left(h_{i j}^{\alpha}\right)^{2}+\frac{\left(\sum_{i=1}^{n} h_{i i}^{\alpha}\right)^{2}}{n-1}-S_{\alpha}\right] .
$$


Note that

$$
\begin{aligned}
\left(\sum_{i=1}^{n} h_{i i}^{\alpha}\right)^{2} & \leq(n-1)\left[\left(h_{11}^{\alpha}+h_{22}^{\alpha}\right)^{2}+\sum_{i>2}\left(h_{i i}^{\alpha}\right)^{2}\right] \\
& =(n-1)\left[\sum_{i=1}^{n}\left(h_{i i}^{\alpha}\right)^{2}+2 h_{11}^{\alpha} h_{22}^{\alpha}\right] .
\end{aligned}
$$

This together with (13) implies

$$
2 h_{11}^{\alpha} h_{22}^{\alpha} \geq \sum_{i \neq j}\left(h_{i j}^{\alpha}\right)^{2}+\frac{\left(\sum_{i=1}^{n} h_{i i}^{\alpha}\right)^{2}}{n-1}-S_{\alpha} .
$$

From the Gauss equation (1) and (14) we get

$$
\begin{aligned}
K(\pi) & =\bar{R}_{1212}+\sum_{\alpha=n+1}^{n+p}\left[h_{11}^{\alpha} h_{22}^{\alpha}-\left(h_{12}^{\alpha}\right)^{2}\right] \\
& \geq \sum_{\alpha=n+1}^{n+p}\left[\sum_{j>2}\left(h_{1 j}^{\alpha}\right)^{2}+\sum_{j>2}\left(h_{2 j}^{\alpha}\right)^{2}+\sum_{j>i>2}\left(h_{i j}^{\alpha}\right)^{2}\right]+\frac{1}{2}\left(\frac{n^{2} H^{2}}{n-1}-S\right)+\bar{K}_{\text {min }} \\
15) & =\frac{1}{2}\left(2 \bar{K}_{\min }+\frac{n^{2} H^{2}}{n-1}-S\right)+\sum_{\alpha=n+1}^{n+p} \sum_{j>i,(i, j) \neq(1,2)}\left(h_{i j}^{\alpha}\right)^{2} .
\end{aligned}
$$

This proves Lemma 4.1.

Lemma 4.2. Let $M^{n}$ be an $n$-dimensional complete submanifold in an $(n+p)$ dimensional Riemannian manifold $N^{n+p}$. If $\sup _{M}\left(S-2 \bar{K}_{\min }-\frac{n^{2} H^{2}}{n-1}\right)<0$, then $M$ is compact.

Proof. From the assumption and Lemma 4.1, it follows that there exists an $\varepsilon>0$ such that $K_{M} \geq \varepsilon$. By the Bonnet-Myers theorem, we know that $M$ is compact. This completes the proof.

Theorem 4.1. Let $\left(M, g_{0}\right)$ be an $n(\geq 4)$-dimensional complete submanifold in an $(n+p)$-dimensional Riemannian manifold $N^{n+p}$. If $\sup _{M}\left[S-\frac{8}{3}\left(\bar{K}_{\min }-\frac{1}{4} \bar{K}_{\max }\right)-\right.$ $\left.\frac{n^{2} H^{2}}{n-1}\right]<0$, then the normalized Ricci flow with initial metric $g_{0}$

$$
\frac{\partial}{\partial t} g(t)=-2 R i c_{g(t)}+\frac{2}{n} r_{g(t)} g(t)
$$

exists for all time and converges to a constant curvature metric as $t \rightarrow \infty$. Moreover, $M$ is diffeomorphic to a spherical space form. In particular, if $M$ is simply connected, then $M$ is diffeomorphic to $S^{n}$.

Proof. By Lemma 4.2, we know that $M$ is compact. When $n \geq 4$, suppose $\left\{e_{1}, e_{2}, e_{3}\right.$, $\left.e_{4}\right\}$ is an orthonormal four-frame and $\lambda \in R$. From the Gauss equation (1) and 
Berger's inequality (2) we have

$$
\begin{aligned}
\left|R_{1234}\right| & =\left|\bar{R}_{1234}+\sum_{\alpha}\left(h_{13}^{\alpha} h_{24}^{\alpha}-h_{14}^{\alpha} h_{23}^{\alpha}\right)\right| \\
& \leq \frac{2}{3}\left(\bar{K}_{\max }-\bar{K}_{\min }\right)+\sum_{\alpha}\left|h_{13}^{\alpha} h_{24}^{\alpha}-h_{14}^{\alpha} h_{23}^{\alpha}\right| .
\end{aligned}
$$

This together with Lemma 4.1 implies

$$
\begin{aligned}
& R_{1313}+\lambda^{2} R_{1414}+R_{2323}+\lambda^{2} R_{2424}-2 \lambda R_{1234} \\
\geq & \left(1+\lambda^{2}\right)\left(2 \bar{K}_{\min }+\frac{n^{2} H^{2}}{n-1}-S\right) \\
& +\sum_{\alpha} \sum_{i<j,(i, j) \neq(1,3)}\left(h_{i j}^{\alpha}\right)^{2}+\sum_{\alpha} \sum_{i<j,(i, j) \neq(2,3)}\left(h_{i j}^{\alpha}\right)^{2} \\
& +\lambda^{2}\left[\sum_{\alpha} \sum_{i<j,(i, j) \neq(1,4)}\left(h_{i j}^{\alpha}\right)^{2}+\sum_{\alpha} \sum_{i<j,(i, j) \neq(2,4)}\left(h_{i j}^{\alpha}\right)^{2}\right] \\
& -2|\lambda|\left[\frac{2}{3}\left(\bar{K}_{\max }-\bar{K}_{\min }\right)+\sum_{\alpha}\left|h_{13}^{\alpha} h_{24}^{\alpha}-h_{14}^{\alpha} h_{23}^{\alpha}\right|\right] \\
\geq & \left(1+\lambda^{2}\right)\left[\frac{8}{3}\left(\bar{K}_{\min }-\frac{1}{4} \bar{K}_{\max }\right)+\frac{n^{2} H^{2}}{n-1}-S\right] \\
& +\sum_{\alpha}\left[\left(h_{24}^{\alpha}\right)^{2}+\lambda^{2}\left(h_{13}^{\alpha}\right)^{2}+\left(h_{14}^{\alpha}\right)^{2}+\lambda^{2}\left(h_{23}^{\alpha}\right)^{2}\right. \\
& \left.-2|\lambda|\left|h_{13}^{\alpha} h_{24}^{\alpha}\right|-2|\lambda|\left|h_{14}^{\alpha} h_{23}^{\alpha}\right|\right] \\
\geq & \left(1+\lambda^{2}\right)\left[\frac{8}{3}\left(\bar{K}_{\min }-\frac{1}{4} \bar{K}_{\max }\right)+\frac{n^{2} H^{2}}{n-1}-S\right] \\
> & 0 .
\end{aligned}
$$

It follows from Theorem $\mathrm{B}$ that $M$ is diffeomorphic to a spherical space form. In particular, if $M$ is simply connected, then $M$ is diffeomorphic to $S^{n}$. This completes the proof of Theorem 4.1.

Proof of Theorem 1.1. By the assumption, we have

$$
\begin{aligned}
S & <\frac{8}{3}\left(\bar{K}_{\min }-\frac{1}{4} \bar{K}_{\max }\right)+\frac{n^{2} H^{2}}{n-1} \\
& \leq 2 \bar{K}_{\min }+\frac{n^{2} H^{2}}{n-1} .
\end{aligned}
$$

So

$$
2 \bar{K}_{\text {min }}+\frac{n^{2} H^{2}}{n-1}-S>0 .
$$

This together with Lemma 4.1 implies $K_{M}>0$.

When $M$ is non-compact, a theorem due to Cheeger-Gromoll-Meyer [9, 11] says that $M$ must be diffeomorphic to $R^{n}$.

When $M$ is compact, we consider the following cases: (i) If $n=2$, it follows from the fact $K_{M}>0$ that $M$ is diffeomorphic to $S^{2}$ or $R P^{2}$. (ii) If $n=3$, Hamilton's theorem [13] shows that $M$ is diffeomorphic to a spherical space form. (iii) If $n \geq 4$, 
the assertion follows from Theorem 4.1.

In particular, if $M$ is simply connected, we conclude that $M$ must be diffeomorphic to $S^{n}$ or $R^{n}$. This completes the proof of Theorem 1.1.

Theorem 4.2. Let $M^{n}$ be an $n(\geq 4)$-dimensional complete submanifold in an $(n+p)$ dimensional simply connected space form $F^{n+p}(c)$ with nonnegative constant curvature c. If $\sup _{M}\left(S-\frac{n^{2} H^{2}}{n-1}\right)<2 c$, then $M$ is diffeomorphic to $S^{n}$.

Proof. It follows from Lemma 4.2 that $M$ is compact. Put

$$
S_{\alpha}:=\sum_{i, j=1}^{n}\left(h_{i j}^{\alpha}\right)^{2}, \quad T_{\alpha}:=\sum_{i=1}^{n} h_{i i}^{\alpha}, \quad \tilde{S}_{\alpha}:=\sum_{i=1}^{n}\left(h_{i i}^{\alpha}\right)^{2} .
$$

Using the same argument as in the proof of Theorem 3.1, we get

$$
\begin{aligned}
& \sum_{k=2}^{n}\left[2\left|h\left(e_{1}, e_{k}\right)\right|^{2}-\left\langle h\left(e_{1}, e_{1}\right), h\left(e_{k}, e_{k}\right)\right\rangle\right]-(n-1) c \\
\leq & \sum_{\alpha}\left[S_{\alpha}-\frac{\tilde{S}_{\alpha}}{n}-\frac{2(n-1)}{n^{2}} T_{\alpha}^{2}+\frac{n-2}{n}\left|T_{\alpha}\right| \sqrt{\frac{n-1}{n}\left(\tilde{S}_{\alpha}-\frac{T_{\alpha}^{2}}{n}\right)}\right]-(n-1) c \\
\leq & \sum_{\alpha}\left[S_{\alpha}-\frac{\tilde{S}_{\alpha}}{n}-\frac{2(n-1)}{n^{2}} T_{\alpha}^{2}+\frac{(n-2)^{2}}{2 n^{2} t} T_{\alpha}^{2}+\frac{(n-1) t}{2 n}\left(\tilde{S}_{\alpha}-\frac{T_{\alpha}^{2}}{n}\right)\right]-(n-1) c,
\end{aligned}
$$

for all $t \in R^{+}$. Taking $t=n-2$, we have

$$
\begin{aligned}
& \sum_{k=2}^{n}\left[2\left|h\left(e_{1}, e_{k}\right)\right|^{2}-\left\langle h\left(e_{1}, e_{1}\right), h\left(e_{k}, e_{k}\right)\right\rangle\right]-(n-1) c \\
\leq & \sum_{\alpha}\left(S_{\alpha}+\frac{n-3}{2} \tilde{S}_{\alpha}-\frac{T_{\alpha}^{2}}{2}\right)-(n-1) c \\
\leq & \frac{n-1}{2} S-\frac{n^{2} H^{2}}{2}-(n-1) c .
\end{aligned}
$$

This together with the assumption implies

$$
\sum_{k=2}^{n}\left[2\left|h\left(e_{1}, e_{k}\right)\right|^{2}-\left\langle h\left(e_{1}, e_{1}\right), h\left(e_{k}, e_{k}\right)\right\rangle\right]-(n-1) c<0 .
$$

It follows from Corollary 2.1 that $\pi_{1}(M)=0$, i.e., $M$ is simply connected.

On the other hand, we see from Theorem 4.1 that $M$ is diffeomorphic to a spherical space form. Therefore, $M$ is diffeomorphic to $S^{n}$. This proves Theorem 4.2.

Theorem 4.3. Let $M^{n}$ be an n-dimensional oriented complete submanifold in an $(n+p)$-dimensional simply connected space form $F^{n+p}(c)$ with nonnegative constant curvature $c$. If $S<2 c+\frac{n^{2} H^{2}}{n-1}$, then $M$ is diffeomorphic to $S^{n}$ or $R^{n}$.

Proof. From Lemma 4.1, we know that $K_{M}>0$. When $M$ is non-compact, the assertion follows from the proof of Theorem 1.1.

When $M$ is compact, we consider the following cases: (i) If $n=2$, from the GaussBonnet theorem we see that the genus of $M$ is zero, and hence $M$ is a topological 
sphere. Therefore, $M$ is diffeomorphic to $S^{2}$. (ii) If $n \geq 3$, it follows from Theorems 3.1 and 4.2 that $M$ is diffeomorphic to $S^{n}$. This completes the proof.

Proof of Theorem 1.2. From Lemma 4.2, we know that $M$ is compact. This together with Theorem 4.3 implies that $M$ is diffeomorphic to $S^{n}$. This completes the proof of Theorem 1.2.

The following example shows that the pinching conditions in Theorems 1.2 and 4.3 are the best possible for arbitrary $n(\geq 2)$ and $p$.

Example 4.1. (i) When $c=0$, let $M:=S^{n-1}\left(\frac{n-1}{n H_{0}}\right) \times R^{1} \subset R^{n+1} \subset R^{n+p}$, where $H_{0}$ is a positive constant. Then $H=H_{0}$ and $S=\frac{n^{2} H^{2}}{n-1}$. (ii) When $c>0$, without loss of generality, we only consider the case $c=1$. Let $M:=S^{1}\left(\frac{1}{\sqrt{1+\lambda^{2}}}\right) \times S^{n-1}\left(\frac{\lambda}{\sqrt{1+\lambda^{2}}}\right) \subset$ $S^{n+1} \subset S^{n+p}$, where $\lambda$ is a positive constant. We have $H=\frac{1}{n}\left[\lambda-(n-1) \frac{1}{\lambda}\right]$ and $S=\lambda^{2}+(n-1) \frac{1}{\lambda^{2}}$. Then $S-\frac{n^{2} H^{2}}{n-1}-2=\frac{(n-2)}{(n-1)} \lambda^{2}$. Thus, for any $\varepsilon>0$ we can find a submanifold $M:=S^{1}\left(\frac{1}{\sqrt{1+\lambda^{2}}}\right) \times S^{n-1}\left(\frac{\lambda}{\sqrt{1+\lambda^{2}}}\right) \subset S^{n+p}$ satisfying $S<2+\frac{n^{2} H^{2}}{n-1}+\varepsilon$.

Proof of Theorem 1.3. By the assumption, we put $I(M):=\varepsilon_{0}>0$. There exists an isometric embedding $\varphi: M \longrightarrow F^{n+p}(c)$ such that

$$
\mu(M, \varphi) \geq \frac{1}{2} \varepsilon_{0}>0 .
$$

Thus $\lambda(M, \varphi)<0$. It follows from Theorem 1.2 that $M$ is diffeomorphic to $S^{n}$. This proves Theorem 1.3 .

The following example shows that Theorem 1.3 is optimal for arbitrary $n(\geq 2)$.

Example 4.2. Let $M:=S^{n-1}\left(\frac{n-1}{n H_{0}}\right) \times R^{1} \subset R^{n+1} \subset R^{n+p}$, where $H_{0}$ is a positive constant. We consider the inclusion $\varphi_{0}: M \longrightarrow R^{n+p}$. Following Example 4.1, we have

$$
\mu\left(M, \varphi_{0}\right)=-\lambda\left(M, \varphi_{0}\right)=0 .
$$

This implies $I(M) \geq I_{0}(M) \geq 0$. Since $M$ is not diffeomorphic to $S^{n}$, it follows from Theorem 1.3 that $I_{0}(M) \leq I(M) \leq 0$. Hence $I(M)=I_{0}(M)=0$.

Finally we present the following differentiable sphere theorem for even dimensional submanifolds in a general Riemannian manifold, which is an extension of Theorems 1.2 and 4.3 as well as the sphere theorems due to Huisken, $\mathrm{Xu}$ and Zhao [14, 25].

Theorem 4.4. Let $M^{n}$ be an even dimensional oriented complete submanifold in an $(n+p)$-dimensional Riemannian manifold $N^{n+p}$. Then

(i) if $S<\frac{8}{3}\left(\bar{K}_{\min }-\frac{1}{4} \bar{K}_{\max }\right)+\frac{n^{2} H^{2}}{n-1}$, then $M$ is diffeomorphic to $S^{n}$ or $R^{n}$.

(ii) if $\sup _{M}\left[S-\frac{8}{3}\left(\bar{K}_{\min }-\frac{1}{4} \bar{K}_{\max }\right)-\frac{n^{2} H^{2}}{n-1}\right]<0$, then $M$ is diffeomorphic to $S^{n}$. 
Proof. (i) It follows from the assumption and Lemma 4.1 that $K_{M}>0$. When $M$ is non-compact, it follows from Cheeger-Gromoll-Meyer's soul theorem $[9,11]$ that $M$ is diffeomorphic to $R^{n}$. When $M$ is compact, it's seen from the assumption and Synge's theorem that $M$ is simply connected. This together with Theorem 1.1 implies that $M$ is diffeomorphic to $S^{n}$. Therefore, we conclude that $M$ is diffeomorphic to $S^{n}$ or $R^{n}$.

(ii) From the assumption and Lemma 4.2, we see that $M$ is compact. This together with (i) implies that $M$ is diffeomorphic to $S^{n}$. This completes the proof of Theorem 4.4 .

\section{Acknowledgement}

The authors would like to thank Professors Simon Brendle, Kefeng Liu, Richard Schoen, Shing-Tung Yau and Fangyang Zheng for their helpful discussions and valuable suggestions.

\section{References}

[1] C. B. Allendoerfer and A. Weil, The Gauss-Bonnet theorem for Riemannian polyhedra, Trans. Amer. Math. Soc., 53(1943), 101-129.

[2] B. Andrews, Positively curved surfaces in the three-sphere, Proc. ICM 2002, Vol.2, Higher Education Press, Beijing, 2002, 221-230.

[3] M. Berger, Riemannian geometry during the second half of the twentieth century, University Lecture Series, Vol.17, American Mathematical Society, Providence, RI, 2000.

[4] S. Brendle, A general convergence result for the Ricci flow in higher dimensions, Duke Math. J., 145(2008), 585-601.

[5] S. Brendle, Ricci Flow and the Sphere Theorem, Graduate Studies in Mathematics, Vol.111, Americam Mathematical Society, 2010.

[6] S. Brendle and R. Schoen, Sphere theorems in geometry, Surveys in Differential Geometry, Vol.13, 2009, 49-84.

[7] S. Brendle and R. Schoen, Manifolds with 1/4-pinched curvature are space forms, J. Amer. Math. Soc., 22(2009), 287-307.

[8] S. Brendle and R. Schoen, Classification of manifolds with weakly 1/4-pinched curvatures, Acta Math., 200(2008), 1-13.

[9] J. Cheeger and D. Gromoll, On the structure of complete manifolds of nonnegative sectional curvature, Ann. of Math., 96(1972), 413-443.

[10] S. S. Chern, A simple intrinsic proof of the Gauss-Bonnet formula for closed Riemannian manifolds, Ann. of Math., 45(1944), 747-752.

[11] D. Gromoll and W. T. Meyer, On complete open manifolds of positive curvature, Ann. of Math., 90(1969), 75-90.

[12] J. Hadamard, Sur certaines propriétés des trajectories en dynamique, J. Math. Pures et Appl., 3(1897), 331-387.

[13] R. Hamilton, Three manifolds with positive Ricci curvature, J. Differential Geom., 17(1982), 255-306.

[14] G. Huisken, Contracting convex hypersurfaces in Riemannian manifolds by their mean curvature, Invent. Math., 84(1986), 463-480.

[15] G. Huisken, Deforming hypersurfaces of the sphere by their mean curvature, Math. Z., 195 (1987), 205-219.

[16] B. Lawson and J. Simons, On stable currents and their application to global problems in real and complex geometry, Ann. of Math., 98(1973), 427-450.

[17] P. Petersen and T. Tao, Classification of almost quarter-pinched manifolds, Proc. Amer. Math. Soc., 137(2009), 2437-2440.

[18] P. Petersen and F. Wilhelm, An exotic sphere with positive sectional curvature, arXiv:math. DG/0805.0812. 
[19] H. E. Rauch, A contribution to differential geometry in the large, Ann. of Math., 54(1951), 38-55.

[20] K. Shiohama, Sphere theorems, Handbook of Differential Geometry, Vol. 1, F.J.E. Dillen and L.C.A. Verstraelen (eds.), Elsevier Science B.V., Amsterdam, 2000.

[21] K. Shiohama and H. W. Xu, The topological sphere theorem for complete submanifolds, Compositio Math., 107(1997), 221-232.

[22] Y. L. Xin, Application of integral currents to vanishing theorems, Scient. Sinica(A), 27(1984), 233-241.

[23] H. W. Xu, Pinching theorems, global pinching theorems and eigenvalues for Riemannian submanifolds, Ph.D. dissertation, Fudan University, 1990.

[24] H. W. Xu, A rigidity theorem for submanifolds with parallel mean curvature in a sphere, Arch. Math., 61(1993), 489-496.

[25] H. W. Xu and E. T. Zhao, Topological and differentiable sphere theorems for complete submanifolds, Comm. Anal. Geom., 17(2009), 565-585.

[26] S. T. Yau and R. Schoen, Lectures on Differential Geometry, Higher Education Press, Beijing, 2004. (in Chinese)

Center of Mathematical Sciences, Zhejiang University, Hangzhou 310027, China

E-mail address: xuhw@cms.zju.edu.cn

Center of Mathematical Sciences, Zhejiang University, Hangzhou 310027, China

E-mail address: gujr@cms.zju.edu.cn 